\title{
LncRNA MT1JP functions as a ceRNA in regulating FBXW7 through competitively binding to miR-92a-3p in gastric cancer
}

Gang Zhang ${ }^{1,2,3 \dagger}$, Shuwei Li ${ }^{2,3 \dagger}$, Jiafei Lu, ${ }^{2,3+}$, Yuqiu Ge ${ }^{2,3}$, Qiaoyan Wang ${ }^{2,3}$, Gaoxiang Ma ${ }^{2,3}$, Qinghong Zhao ${ }^{4}$, Dongdong Wu', Weida Gong ${ }^{5}$, Mulong Du, ${ }^{2,3}$, Haiyan $\mathrm{Chu}^{2,3}$, Meilin Wang ${ }^{2,3}$, Aihua Zhang ${ }^{6^{*}}$ and Zhengdong Zhang ${ }^{2,3^{*}}$

\begin{abstract}
Background: Emerging evidence has shown that dysregulation function of long non-coding RNAs (IncRNAs) implicated in gastric cancer (GC). However, the role of the differentially expressed IncRNAs in GC has not fully explained.

Methods: LncRNA expression profiles were determined by IncRNA microarray in five pairs of normal and GC tissues, further validated in another 75 paired tissues by quantitative real-time PCR (qRT-PCR). Overexpression of IncRNA MTIJP was conducted to assess the effect of MTIJP in vitro and in vivo. The biological functions were demonstrated by luciferase reporter assay, western blotting and rescue experiments.

Results: LncRNA MT1JP was significantly lower in GC tissues than adjacent normal tissues, and higher MT1JP was remarkably related to lymph node metastasis and advance stage. Besides, GC patients with higher MT1JP expression had a well survival. Functionally, overexpression of IncRNA MT1JP inhibited cell proliferation, migration, invasion and promoted cell apoptosis in vitro, and inhibited tumor growth and metastasis in vivo. Functional analysis showed that IncRNA MT1JP regulated FBXW7 expression by competitively binding to miR-92a-3p. MiR-92a-3p and down-regulated FBXW7 reversed cell phenotypes caused by IncRNA MT1JP by rescue analysis.

Conclusion: MT1JP, a down-regulated IncRNA in GC, was associated with malignant tumor phenotypes and survival of GC. MTIJP regulated the progression of GC by functioning as a competing endogenous RNA (ceRNA) to competitively bind to miR-92a-3p and regulate FBXW7 expression. Our study provided new insight into the post-transcriptional regulation mechanism of IncRNA MT1JP, and suggested that MT1JP may act as a potential therapeutic target and prognosis biomarker for GC.
\end{abstract}

Keywords: IncRNA, MT1JP, Gastric cancer, ceRNA, Prognosis

\footnotetext{
* Correspondence: aihuagzykd@163.com; drzdzhang@gmail.com

${ }^{\dagger}$ Equal contributors

${ }^{6}$ Key Laboratory of Environmental Pollution Monitoring and Disease Control, Ministry of Education, Guizhou Medical University, Guiyang 550025, Guizhou, China

${ }^{2}$ Department of Environmental Genomics, School of Public Health, Jiangsu Key Laboratory of Cancer Biomarkers, Prevention and Treatment, Collaborative Innovation Center for Cancer Personalized Medicine, Nanjing Medical University, 101 Longmian Avenue, Jiangning District, Nanjing 211166, China

Full list of author information is available at the end of the article
} 


\section{Background}

Recently, high-throughput genome and transcriptome sequencing and microarrays have indicated that apart from protein-coding genes, $75 \%$ of the human genomes is transcribed into noncoding RNAs [1, 2]. LncRNAs are functionally catalogued as noncoding transcripts are more than 200 nucleotides in length, and have no potential protein-coding ability. The Encyclopedia of DNA Elements (ENCODE) Project Consortium revealed that more than 28,000 lncRNAs were transcribed in the whole genomes [2]. The aberrant expression and deficiency or mutation of lncRNAs were reported to be involved in numerous complex diseases, including cancers $[3,4]$. Mounting evidence indicated that lncRNAs are implicated in a variety of biological processes, including chromatin interaction, transcription regulation, mRNA post-transcriptional regulation and epigenetic regulation [5-7].

Additionally, increasing experimental evidence supports that lncRNA functions as competitive endogenous RNA (ceRNA), which compete for microRNA (miRNA) to up-regulate the expression of a target gene. The ceRNA hypothesis provide new insights into the function of a large amount of uncharacterized lncRNAs [8]. It has been reported that muscle-specific long noncoding RNA (linc-MD1) regulate MAML1 and MEF2C expression by sponging miR-133 and miR-135 [9]. Another study has shown that lncRNA BC032469 acted as a ceRNA for miR-1207-5p to up-regulate the expression of hTERT and promoted proliferation in gastric cancer (GC) [10].

GC is the fourth most frequent malignancy and contributes to the second leading cause of cancer mortality. Although effective medical treatments such as surgery, chemotherapy and radiation have been improved, GC patients are usually diagnosed with advanced stage, resulting in a low five-year survival rate [11-13]. Currently, GC is still a globe health problem, which highlights the need for further studies of molecular mechanism of GC and identify effective therapeutic targets. Emerging evidence have shown that aberrant expression of many lncRNAs were observed in gastric cancer and significantly associated with carcinogenesis, diagnosis and prognosis of gastric cancer $[14,15]$. However, the role of lncRNA and its molecular mechanism involved in GC remain largely obscure. To systematically identify lncRNAs involved in the carcinogenesis of $\mathrm{GC}$, we analyzed and integrated the results of our lncRNA microarray and Gene Expression Omnibus (GEO) database. Among the deregulated lncRNAs, we selected and investigated MT1JP, a lncRNA located at $16 q 12.2$ region. Here, we found that lncRNA MT1JP acted as a competing endogenous RNA in regulating FBXW7 through sponging miR-92a-3p and inhibit cell proliferation, migration, invasion and promote cell apoptosis.

\section{Methods}

\section{GC tissues}

A total of 80 pairs of matched normal and GC tissues were collected from The Second Affiliated Hospital of Nanjing Medical University between February 2009 and October 2013. Five paired adjacent normal tissues and GC tissues were randomly selected in lncRNA microarrays study, and the remaining 75 paired gastric tissues were applied to qRT-PCR analysis. Additionally, another 330 paraffin-embedded GC tissues and corresponding follow-up information were obtained from Nantong Tumor Hospital between February 2008 and March 2013. All subjects have written informed consent and this study was approved by the Institutional Review Boards of Nanjing Medical University.

\section{LncRNA microarrays}

The lncRNA expression characteristics of GC were investigated by Arraystar Human LncRNA microarray V2. 0 , which contains 30,215 coding genes and 33,045 lncRNAs collected from several databases such as UCSC, Ensembl, RefSeq and the lncRNAs reported from literatures were also included. The microarray and data collection were conducted by KangChen Bio-tech (Shanghai, PR China). The details are as mentioned previously [16]. In addition, non-coding RNA profiling GSE53137 from the same platform was downloaded from GEO database, which investigate lncRNAs expression in six pairs of human gastric adenocarcinoma and adjacent normal tissues. Paired $t$-test was conducted to assess the differentially expressed lncRNAs between tumor and adjacent normal tissues (fold change $>2.0$ and $P$ value $<0.05)$.

\section{qRT-PCR analysis}

The total RNA from GC tissue or cell lines were extracted using Trizol Reagent (Invitrogen, CA, USA) and mirVana miRNA Isolation Kit (Applied Biosystems) according to the manufacturer's instructions. M-MLV reverse transcriptase (Invitrogen) was used for lncRNA MT1JP reverse transcription. The expression of lncRNA MT1JP and FBXW7 was detected by ABI 7900HT RealTime PCR System (Applied Biosystem, Foster City, CA, USA), using SYBR Green assays (TaKaRa Biotechnology, Dalian, China) and GAPDH was used as the internal control. The expression of miR-92a-3p was measured using TaqMan MicroRNA Assays (Applied Biosystems) and U6 was treated as an internal control. All the primer sequences were available in Additional file 1: Table S2.

\section{LncRNA coding capacity prediction}

Coding Potential Assessment Tool (CPAT, http://lilab.re search.bcm.edu/cpat/) was used to assess the coding capacity of lncRNA MT1JP. The CPAT conducted a logistic 
regression model by using the sequence features of open reading frame coverage, open reading frame size, hexamer usage bias and Fickett TESTCODE statistic. The CPAT chose 0.364 as a cutoff as human coding probability (CP). $\mathrm{CP}<0.364$ suggests noncoding sequence, whereas $\mathrm{CP} \geq$ 0.364 indicates coding sequence [17].

\section{Nuclear-cytoplasmic fractionation}

Nuclear/cytoplasmic fractionation was conducted by the Protein and RNA Isolation System (Ambion) according to the manufacturer's protocols. U6 was treated as a nuclear control while GAPDH was a cytoplasmic control.

\section{Cell proliferation, migration, invasion, apoptosis and cell cycle analysis}

The Cell Counting Kit 8 (Dojindo) was used to measure cell viability. The spectrophotometric absorbance at $450 \mathrm{~nm}$ for each sample was detected using spectrophotometer Infinite M200 (Tecan). All the experiments were repeated three times in six replicates. The transwell assay was used to evaluate cell migration. Cell invasion was assessed using BioCoat Matrigel Invasion Chamber (BD Biosciences Discovery Labware). Cell numbers for cell migration and invasion in three random fields were counted. Cells were stained by Annexin $\mathrm{V}$ and propidium iodide using the Annexin V-FITC Apoptosis Detection kit (Invitrogen), and the percentage of apoptosis was examined with flow cytometry (BD Bioscience, San Jose, CA, USA). For detection of cell cycle, cells were stained with PI after 48 h transfection and examination were performed by FACS Calibur system (Beckman Coulter).

\section{Luciferase reporter assay}

The full-length lncRNA MT1JP cDNA was cloned into the BamHI and Xhol enzyme restriction sites of psiCHECK-2 vector (Promega) (psicheck-2-MT1JPwild vector). The potential miR-92a-3p binding sites were mutated by the QuikChang site-directed mutagenesis kit (Agilent Technologies) (psicheck-2-MT1JPmut vector). The psicheck-2-MT1JP-wild vector or psicheck-2-MT1JP-mut vector and miR-92a-3p mimics were co-transfected into BGC-823 and SGC-7901 cell by Lipofectamine 2000 (Invitrogen, Carlsbad, CA, USA). The wild and mutant miR-92a-3p was cloned into the BamHI and XhoI enzyme restriction sites of psiCHECK-2 vector (Promega). miR-92a-3p-wild or miR-92a-3p-mutant and lncRNA MT1JP overexpression vector were co-transfected into both SGC-7901 and BGC-823 cell by Lipofectamine 2000 (Invitrogen, Carlsbad, CA, USA). The luciferase activity was assessed by Dual-Luciferase Reporter Assay System (Promega, Madison, WI, USA) and the firefly luciferase activity was normalized by renilla luciferase activity.

All the cloned sequences were validated by DNA sequencing.

\section{Tumor formation test}

Twenty (10 nude mice in each group) five-week-old female athymic BALB/c nude mice were kept under pathogen-free conditions. BGC-823 cells that were transfected with an empty vector and the MT1JP vector were collected and resuspended at a concentration of 1 . $5 \times 10^{7}$ cells $/ \mathrm{mL}$. Then, $0.1 \mathrm{~mL}$ of the cells that were transfected with the MT1JP vector and an empty vector were subcutaneously injected into each posterior flank of the nude mice. The tumor weights and volumes were examined every 3 days. The mice were killed after 16 days post-injection, and the tumors from the mice were measured.

\section{Immunohistochemistry (IHC)}

Hematoxylin and eosin ( $\mathrm{H} \& \mathrm{E})$ staining was applied to select representative areas. The antibodies against $\mathrm{Ki}$ 67 (Abcam, Cambridge, MA, USA) were applied for IHC. The process of IHC was according to our previous study [18].

\section{Western blotting analysis}

Protein was isolated from BGC-823 and SGC-7901 cell lines as previously stated [19].

Forty micrograms of total protein s were run on a 14 . $7 \%$ polyacrylamide gel, and then transferred to polyvinylidene difluoride membranes (Hybondenhanced chemiluminescence; Amersham Pharmacia Biotech). The antibodies against FBWX7(1:1000) and $\beta$-actin (1:1000) were purchased from Abcam (Abcam, Cambridge, MA, USA). The antibodies against Caspase-9 antibody (1: 1000) and Caspase-3 (1:1000) were purchased from Cell Signaling Technology (Cell Signaling Technology, USA). The protein was measured with a Phototope-horseradish peroxidase Western blot detection kit (Cell Signaling Technology, Inc.), and $\beta$-actin was treated as an internal control.

\section{Statistical analysis}

For continuous variables, the results were shown as mean \pm SD. Student's $t$-test was applied to compare the difference of means between two groups. Differentially expressed MT1JP between gastric cancer and normal tissues from TCGA database was also evaluated by Student's $t$-test. We used Kaplan-Meier curve and log-rank test to evaluate the effect of lncRNA MT1JP on survival of GC patients. The relationship between lncRNA MT1JP and FBXW7 expression was assessed by Spearman Pearson correlation analysis. A 
two-sided $P$ value $<0.05$ was considered as statistically significant. All analyses were performed using SAS software (version 9.2; SAS Institute, Inc., Cary, NC, USA).

\section{Results}

Differently expressed IncRNA identified in microarray and GEO database

LncRNA microarray was conducted in five pairs of GC tissues and adjacent normal tissues to investigate the lncRNA expression signatures of GC. Compared with normal tissues, 551 lncRNAs were up-regulated and 278 lncRNAs were down-regulated in GC tissues (fold change $>2.0$ and $P<0.05$ ). In order to reduce false positive, GSE53137, IncRNA profile based on the same microarray platform as our study, was download from GEO database. We integrated and selected the consistently differently expressed lncRNAs in both GSE53137 database and our microarray. The top 10 significantly up-regulated and down-regulated candidate lncRNAs are shown in Additional file 1: Table S1. Given that among the differently expressed lncRNAs, the fold change of
MT1JP was the most remarkable, therefore, we focused on lncRNA MT1JP in the further study.

\section{Characterization of IncRNA MT1JP in GC}

Subsequently, the expression levels of lncRNA MT1JP were explored in a large-cohort of 75 pairs of GC tissues and corresponding adjacent normal tissues. Significantly lower MT1JP expression was observed in GC tissues than in adjacent normal tissues $(P<0.001$, Fig. 1a).Similar result was also acquired from The Cancer Genome Atlas (TCGA) database $(P=0.018$, Additional file 1: Figure S1). We then examined the clinic pathological role of lncRNA MT1JP, and found that higher MT1JP expression was significantly related to lymph node metastasis and advance stage $(P=0.020$ and 0.013 , respectively, Fig. $1 \mathrm{~b}$ and $\mathrm{c})$. We further investigate the association between IncRNA MT1JP and survival of 308 GC patients with follow-up data. KaplanMeier curve and log-rank results indicated that GC patients with higher lncRNA MT1JP expression had a well prognosis and longer survival $(H R=1.33$, $95 \% \mathrm{CI}=1.02-1.76, \log$-rank $P=0.031$, Fig. $1 \mathrm{~d})$.
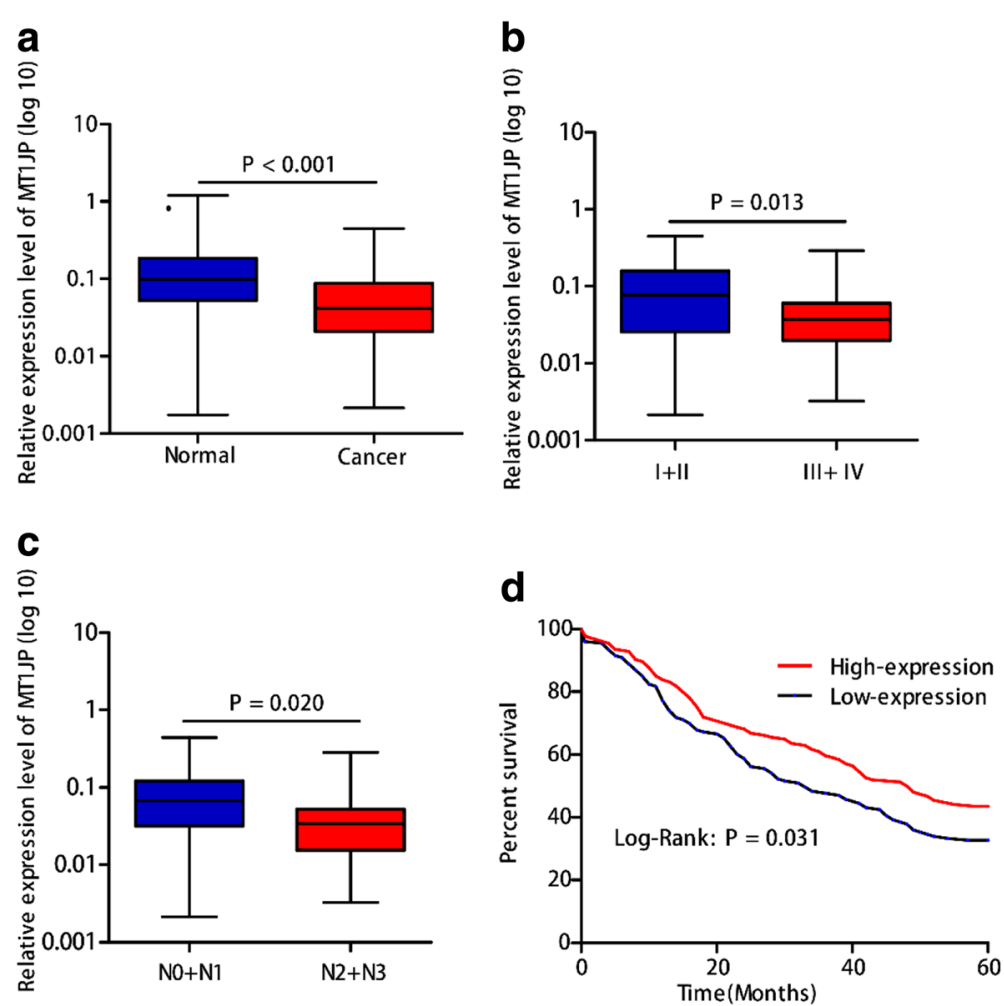

Fig. 1 LnCRNA MTIJP was significantly up-regulated in GC tissue and associated with progression and prognosis of GC. a Relative expression level of IncRNA MTIJP in GC tissue and adjacent normal tissues. b Relative expression level of IncRNA MT1JP in GC patient with early stage (I/II) and advanced stage (III/IV). c Differential expression levels of IncRNA between GC patients with or without lymph node metastasis. $\mathbf{d}$ Kaplan-Meier curve showing survival in GC patients stratified by IncRNA MT1JP expression. Patients were divided into high (red) and low expression group (blue) according to the median of IncRNA MT1JP expression 
LncRNA MTIJP overexpression inhibited cell proliferation, migration and invasion and promoted cell apoptosis in vitro

Consider that lncRNA MT1JP was down-regulated in GC tissues, we next investigated the effects of lncRNA MT1JP overexpression on GC cell phenotypes.

Because the expression of MT1JP was relatively lower in SGC-7901 and BGC-823 cells, we performed overexpression experiments in these two cell lines (Additional file 1: Figure S1B). Constructed pEGFPN1 vector containing full-length lncRNA MT1JP was transfected into SGC-7901cell and BGC-823 cell, and found that the expression was effectively up-regulated as compare with negative control (NC) (Fig. 2a). Intriguingly, lncRNA MT1JP overexpression markedly inhibit cell proliferation in both SGC-7901and BGC823 cells (Fig. 2b). Moreover, cell migration and invasion abilities in both GC cell lines were also significantly suppressed by lncRNA MT1JP overexpression (Fig. 2c and d). Flow cytometry analysis demonstrated that compared with NC, IncRNA MT1JP overexpression prominently promoted cell apoptosis in GC cells (Fig. 2e and $\mathrm{f}$ ).

\section{LncRNA MT1JP overexpression inhibited tumor growth and metastasis in vivo}

BGC-823 cells with MT1JP overexpression or negative control were subcutaneously injected into the back flank of nude mice. An effective higher expression level of MT1JP was observed in MT1JP overexpression group than that in negative control group. In line with in vitro analysis, the results indicated that the volume and weight of xenograft tumor were significantly lower in MT1JP overexpression group as compared with negative control group (Fig. 3a-e). Furthermore, H\&E and immunohistochemistry for Ki67 was performed to detect the expression of Ki67, and results showed that MT1JP overexpression resulted in a substantial reduce of Ki67 protein expression (Fig. 3f). We also detected the expression of apoptosis mediators by western blot. The

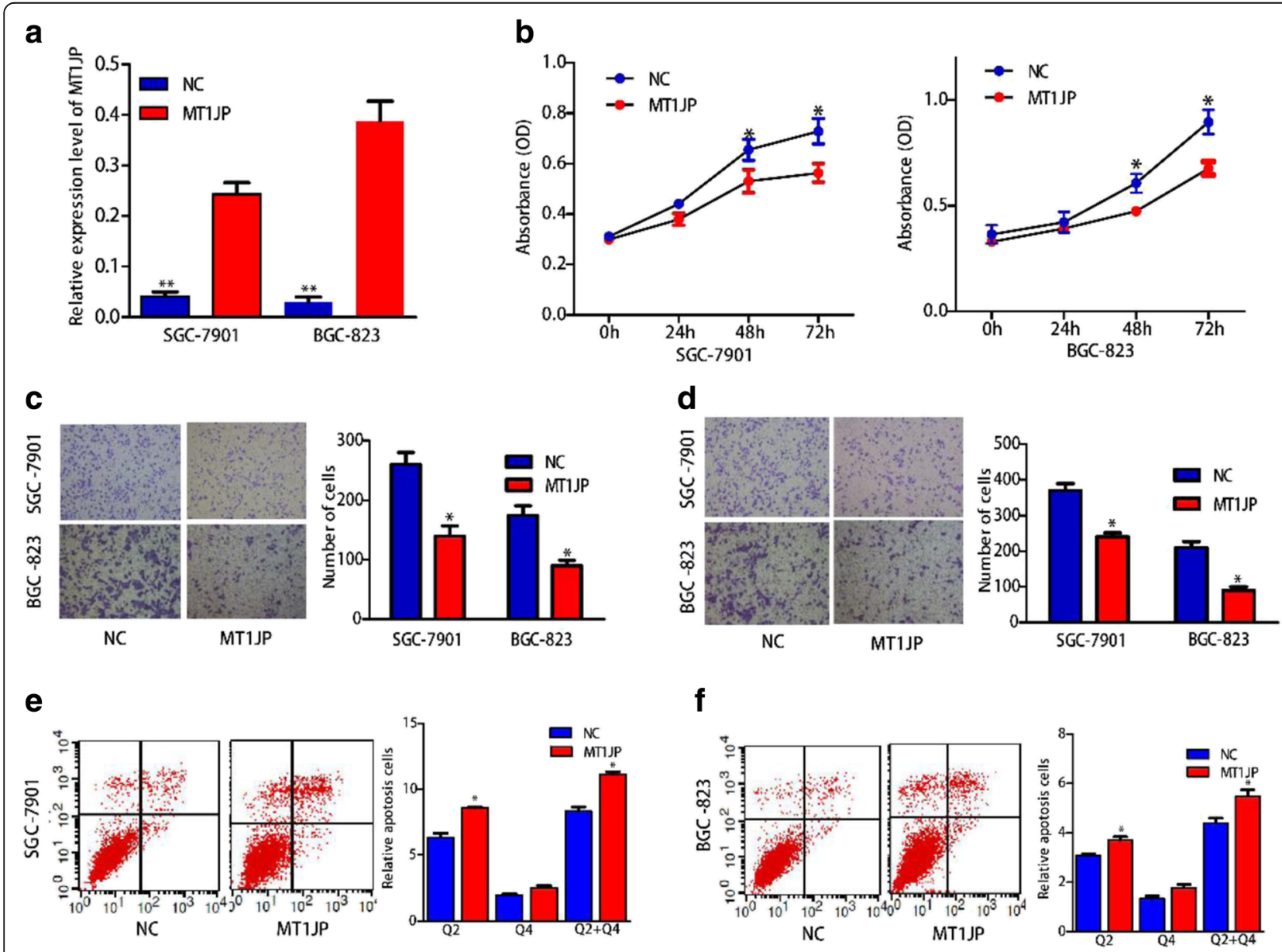

Fig. 2 LnCRNA MT1JP inhibited GC cell proliferation, migration, invasion and promoted cell apoptosis. a Effect of IncRNA MT1JP overexpression on proliferation of GC cell lines. b, c Overexprssion of InCRNA MT1JP significantly inhibited migration and invasionof GC cell. d Overexprssion of IncRNA MT1JP significantly enhancedapoptosis of GC cell. MT1JP induces the apoptosis of SGC7901 (e) and MGC-823 (f) 
a

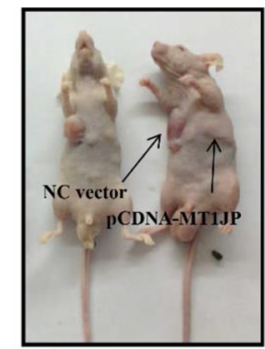

d

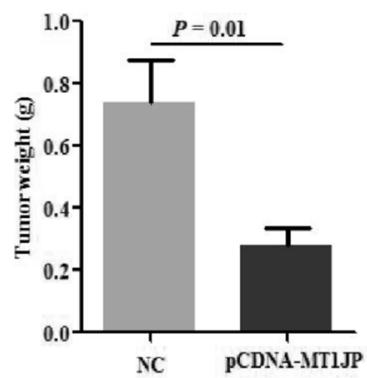

b

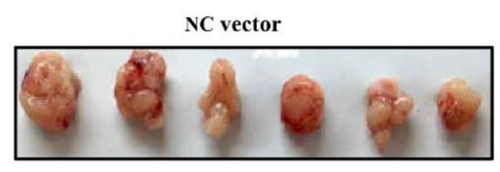

pCDNA-MT1JP

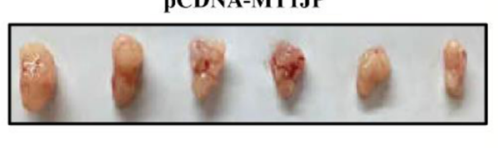

e

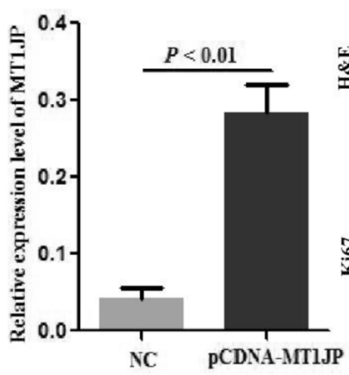

C

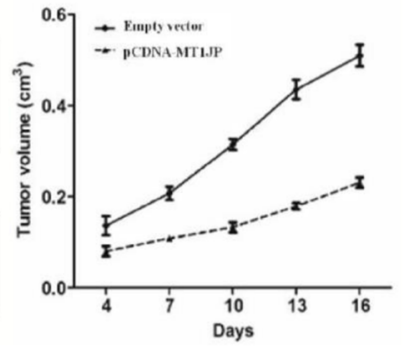

f

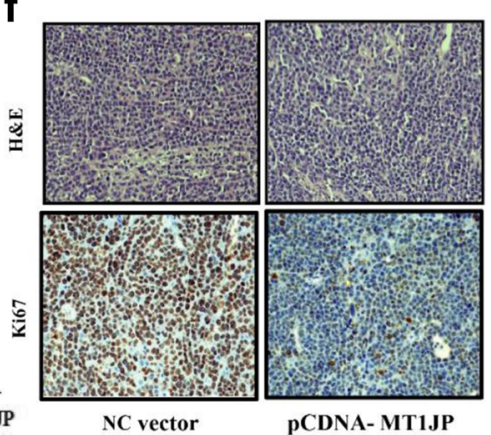

Fig. 3 Xenograft tumor formation of GC cell with overexpression of IncRNA MT1JP in nude mice. a-d Overexpression of IncRNA MT1 JP inhibited the growth of transplanted BGC-823 GC cell in nude mice. e Relative expression level of MT1JP of xenograft was significantly upregulated in the MT1JP overexpression group compared with NC group. $\mathbf{f}$ The xenografts were H\&E stained and expression of ki67 were measured by immunohistochemistry

results indicated that overepresion of MT1JP was observedcorrelated with the increased cleaved caspase 3 and cleaved caspase 9 (Additional file 1: Figure S2).

\section{LncRNA MT1JP played a ceRNA role in regulating FBXW7 expression by binding to miR-92a-3p}

The coding capability prediction of MT1JP was determined by online tool CPAT, and the result indicated that MT1JP had no apparent protein-coding ability (Additional file 1: Figure S3A). We next conducted nuclear-cytoplasmic fractionation to evaluate the lncRNAMT1JP subcellular localization, and found that lncRNA MT1JP was primarily detected in the cytoplasm of SGC-7901 and BGC-823 cells (Additional file 1: Figure S3B). To identify the potential miRNA targets of lncRNA MT1JP, in silico analysis was performed by using both FINDTAR3 (http://bio.sz.tsin ghua.edu.cn/) and RegRNA (http://regrna.mbc.nctu.edu.tw/ index1.php) databases, and jointly predicted that seven miRNAs (miR-24, miR-107, miR-195, miR-298, miR-185, miR-92a-3p and miR-223-5p) may act as biological targets of lncRNA MT1JP (Fig. 4a). We then conducted luciferase reporter assay to validate the binding of candidate miRNAs with IncRNA MT1JP, and selected miR-92a-3p with the mostly decreased luciferase activity into the further study (Fig. 4b). The expression levels of miR-92a-3p was significantly higher in GC tissues than in adjacent normal tissues $(P<0.01$, Fig. $4 c)$. In addition, miR-92a-3p mimic prominently decreased luciferase activity in MT1JP-wild not in MT1JP-mut in both SGC-7901 and BGC-823 cell lines, which indicated that miR-92a-3p is a target of IncRNA MT1JP (Fig. 4d). Further prediction of target genes of miR92a-3p was performed by TargetScan (http://www.targets can.org/vert_71/) and microRNA.org (http://www.micro rna.org/microrna/home.do), and FBXW7 was selected as the target gene of miR-92a-3p for it acquired consistently highest score in the prediction analysis of both softwares. Luciferase reporter results showed that remarkably reduced luciferase activity was observed in FBXW7-wild not in FBXW7-mut (Fig. 5a). We used RT-PCR to assess the RNA expression of miR-92a-3p and FBXW7 in cells overexpressed MT1JP. The results indicated that the RNA level of FBXW7 significantly increased while no significant change in miR-92a-3p (Additional file 1: Figure S5). Moreover, overepresion of MT1JP markedly declined luciferase activity in miR-92a-3p-wild not in miR-92a-3p-mutant in both SGC-7901 and BGC-823 cell lines (Additional file 1: Figure S4A). Additionally, we detected the expression of miR-92a3p correlate with MT1JP and FBXW7 in clinical samples. The results showed miR-92a-3p reversely correlate with FBXW7, but not correlate with MT1JP (Additional file 1: Figure S6).

We also found significantly lower expression of FBXW7 in GC tissues, as compared with corresponding normal tissues $(P<0.01$, Fig. $5 b)$. Moreover, a positive correlation between MT1JP and FBXW7 expression was detected in GC tissues $(r=0.452, P<0.001$, Fig. $5 \mathrm{c})$. The western blotting and RT-PCR analysis results showed that miR-92a-3p mimic reduced the expression of 


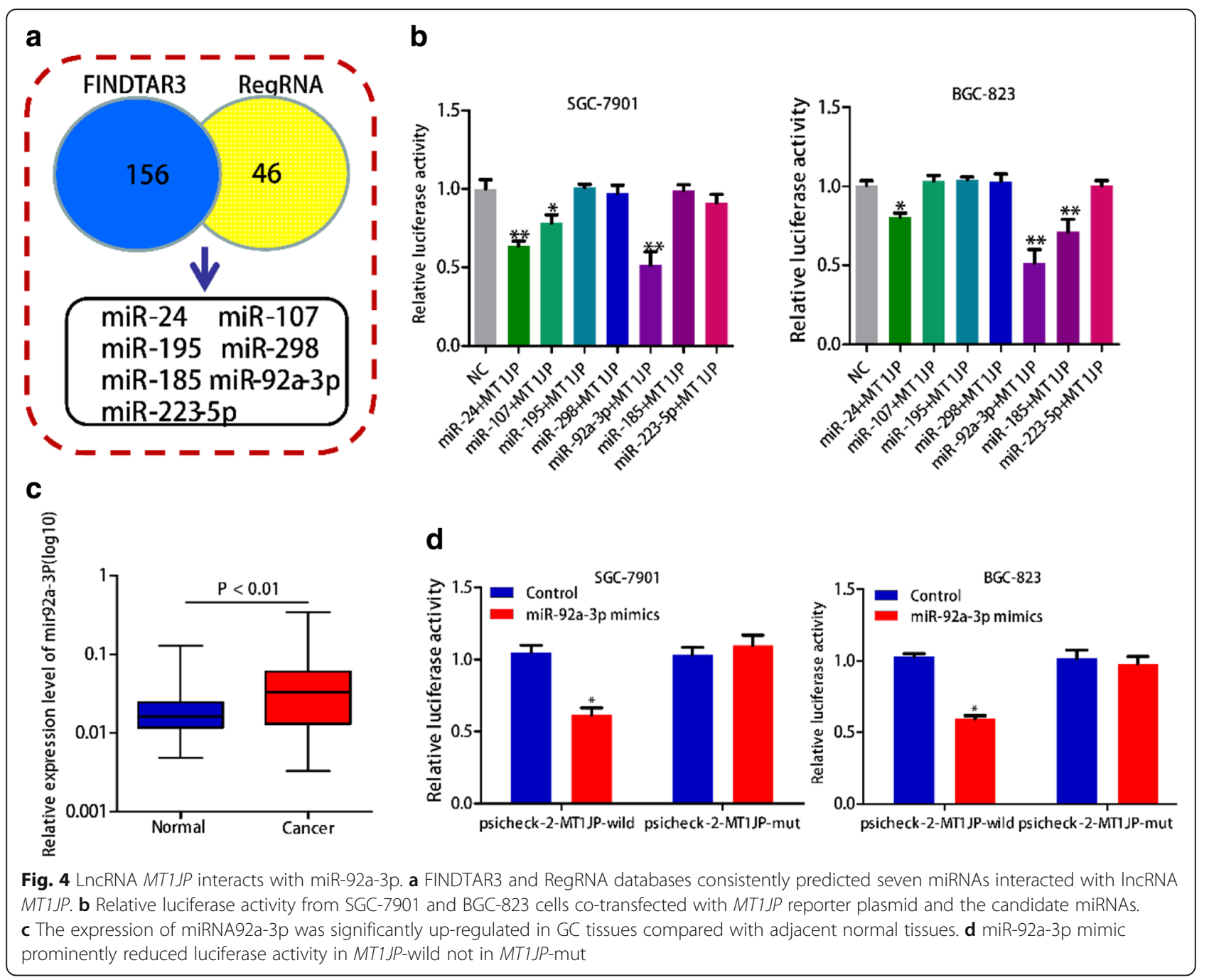

FBXW7, whereas lncRNA MT1JP overexpression substantially abolished this effect. Additionally, lncRNA MT1JP had no up-regulated effect on FBXW7 with miR92a-3p inhibitor (Fig. 5d) (Additional file 1: Figure S4B).

\section{MiR-92a-3p mimics and si-FBXW7 reversed the} suppression function of IncRNA MT1JP in vitro

Since lncRNA $M T 1 J P$ played a ceRNA role in regulating $F B X W 7$ expression by binding to miR-92a-3p, we performed rescue assays to validate whether miR-92a-3p and FBXW7 were involved in the lncRNA MT1JP-mediated inhibition proliferation, migration and invasion and promoting apoptosis in GC cells. To determine the effects of miR-92a-3p and FBXW7 in GC cell phenotypes, BGC-823 cell line was transfected with miR-92a$3 \mathrm{p}$ mimics and si-FBXW7, respectively. For rescue experiment, lncRNA $M T 1 J P$ overexpression vector, miR92a-3p mimics and si-FBXW7 were co-transfected into BGC-823 cell. Intriguingly, both miR-92a-3p mimics and si-FBXW7 remarkably promoted cell prolifereation, migration and invasion, and decreased cell apoptosis, respectively (Fig. 6). Co-transfection group (lncRNA MT1JP overexpression vector, miR-92a-3p mimics and si- $F B X W 7$ ) could reversed the decrease on cell proliferation caused by lncRNA MT1JP overexpression (Fig. 6a). Moreover, flow cytometry analysis revealed that miR92a-3p mimics and si- $F B X W 7$ significantly suppress apoptotic cell rate caused by lncRNA MT1JP overexpression (Fig. 6b-c). Cell migration and invasion experiments revealed that miR-92a-3p mimics and si-FBXW7 prominently reversed the effects of lncRNA MT1JP overexpression (Fig. 6d-f).

\section{Discussion}

There has been wide consensus that IncRNA play a key role in a variety of biological process, and aberrant expression or function of lncRNAs are commonly observed in a variety of cancers, including GC $[2,20]$. Recently, lncRNA microarray was widely performed to investigate the lncRNA expression signatures, and identified significantly 

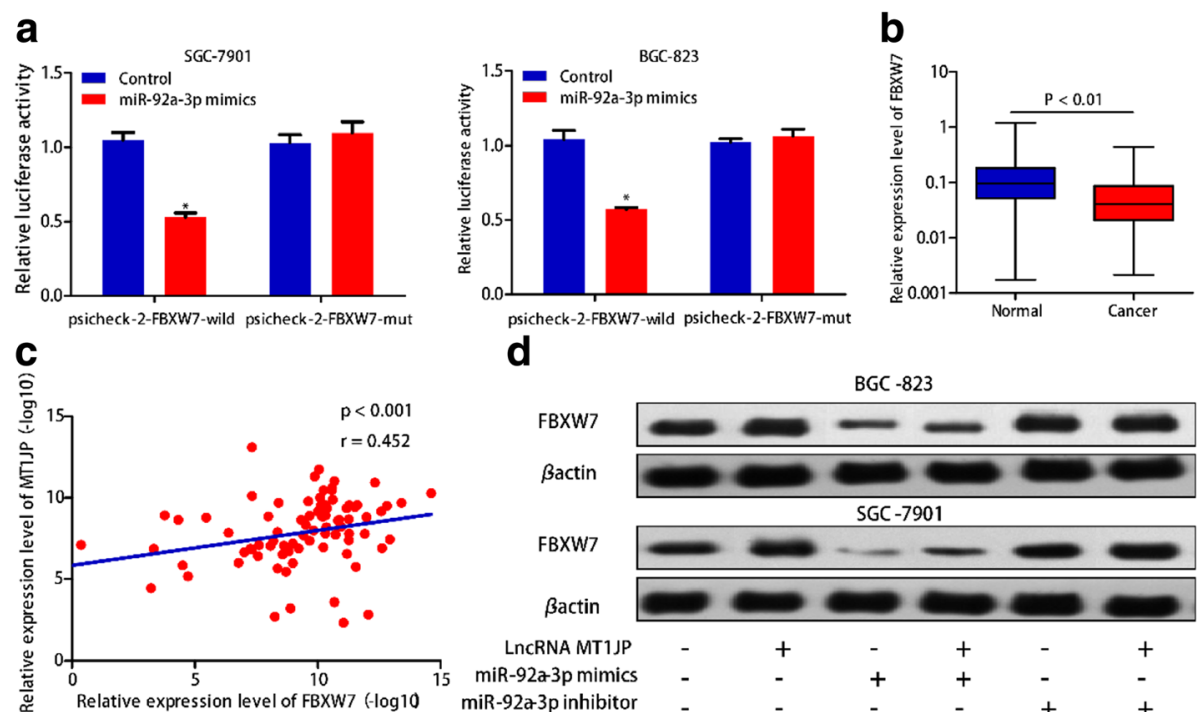

d

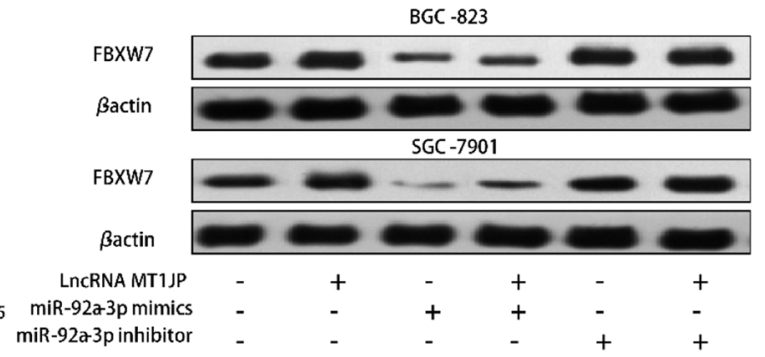

Fig. 5 LncRNA MT1JP regulate FBXW7 expression by sponging miR-92a-3p. a miR-92a-3p significantly reduced luciferase activity in FBXW7-wild not in FBXW7-mut. b Compared with adjacent normal tissues, FBXW7 was remarkably upregulated in GC tissue. c Spearman-Pearson correlation between IncRNA MT1JP and FBXW7 expression in GC tissues (Spearman $r=0.452, P<0.001$ ). d Western blotting analysis of FBXW7 in BGC-823 and SGC-7901 cells with overexpression of IncRNA MT1JP and/or miR-92a-3p mimics and/or miR-92a-3p inhibitor

altered lncRNAs in cancers. In this study, we applied lncRNA microarray and integrated a GEO dataset to depict the lncRNA profiles, and found that MT1JP was the most significantly deregulated lncRNA with the largest Fold change. In the further validation study, the results showed that lncRNA MT1JP was significantly downregulated in GC tissues compared with adjacent normal tissues. In line with the our results, a previous study applied an mRNA/lncRNA microarray in 76 pairs of tumor and normal tissue sample from live, colon, lung and gastric cancer, and revealed that lncRNA MT1JP had remarkably lower expression in all tumor samples [21]. Furthermore, our study found that expression of lncRNA MT1JP was prominently associated with lymph node metastasis and advance stage, suggesting a clinic pathological role of lncRNA MT1JP in GC. We also detected the involvement of miR-92 and FBXW7 in disease stage and progression. The results showed miR-92 and FBXW7 were associated with the TNM stage (Additional file 1: Figure S7 and S8). In addition, log-rank results demonstrated that GC patients with higher expression of IncRNA MT1JP had a well prognosis. These findings suggested that IncRNA MT1JP may act as a promising prognosis biomarker for GC. Additionally, we employed the ROC curve to calculate the prognostic significance of combination of MT1JP and miR-92a. The result indicated combination of MT1JP and miR-92a could improve the predictive value for prognosis of GC patient (Additional file 1: Figure S9).

To further explore the effect of MT1JP on cell phenotypes, MT1JP overexpression vector was constructed and transfected into SGC-7901and BGC-823 cells.
Compared with negative control, lncRNA MT1JP overexpression significantly inhibited cell proliferation, migration and invasion and promoted cell apoptosis in both SGC-7901 cell and BGC-823 cell. Consistent with these findings in vivo, animal experimental study also confirmed that lncRNA MT1JP overexpreesion inhibited tumor growth and metastasis. Intriguing, Liu et al. reported that knockdown of MT1JP increased cell proliferation, migration and invasion while suppressed cell apoptosis in live cell line [21]. These results suggest a tumor-suppressor role of lncRNA MT1JP in GC and highlight the need for further study of the molecular mechanism of MT1JP involving in GC.

Nuclear-cytoplasmic fractionation were conducted to assess the subcellular localization and showed that majority of lncRNA MT1JP were located in cytoplasmic.

Emerging evidence support the ceRNA hypothesis, and indicated that ceRNA regulation implicated in the carcinogenesis of GC $[22,23]$. It has been reported that IncRNA HOTAIR may act as a ceRNA to spong miR331-3p and regulate the expression of HER2 in GC [24]. Therefore, we speculated that lncRNA may function as a ceRNA, participate in carcinogenesis of GC. Bioinformatics analysis and luciferase reporter assay confirmed that lncRNA MT1JP is a target of miR-92a-3p. Furthermore, our functional analysis also revealed that FBXW7 is a direct target of miR-92a-3p.

Given that both lncRNA MT1JP and FBXW7 interact with miR-92a-3p, suggesting that lncRNA MT1JP may regulate FBXW7 expression by competing bind to miR92a-3p. In this study, the correlation analysis results 
a
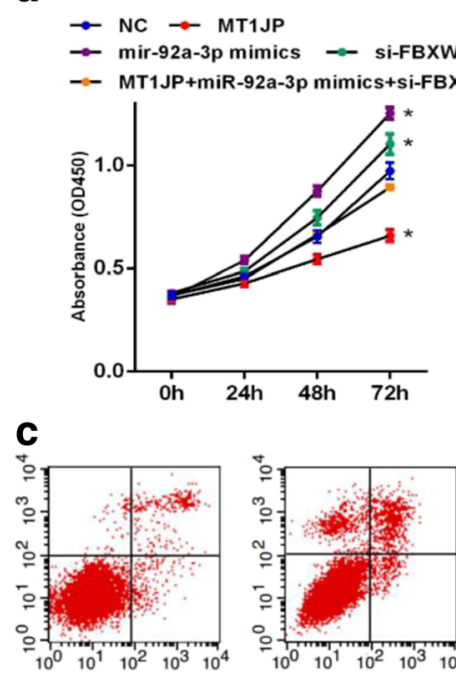

NC

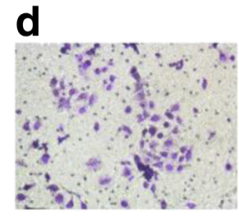

NC

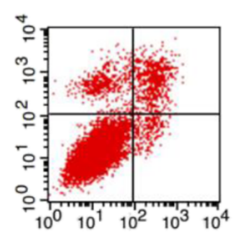

MT1JP

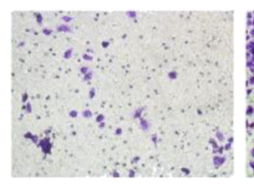

MT1JP

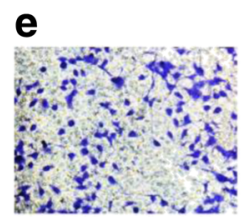

NC

\section{f}

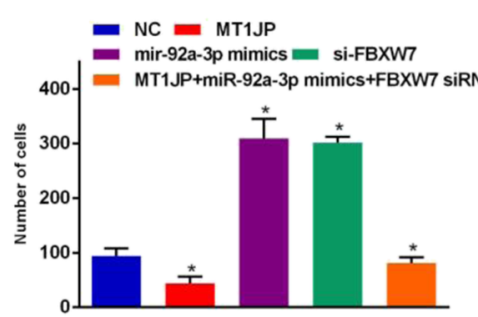

b
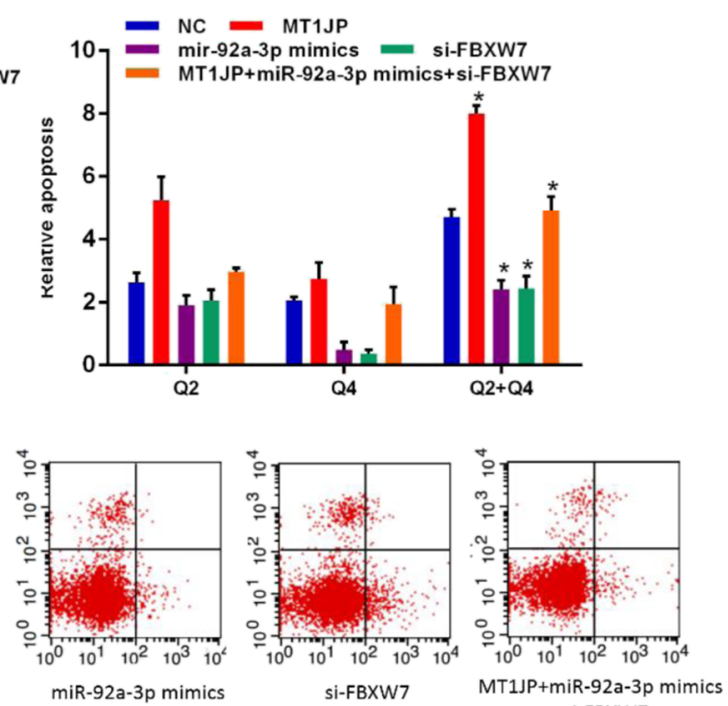

+ si-FBXW7

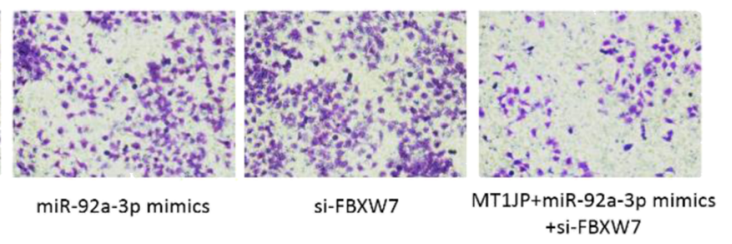

Fig. 6 MiR-92a-3p mimics and si-FBXW7 reversed the suppression function of IncRNA MT1JP in vitro, a Effect of MiR-92a-3p mimics, si-FBXW7, and IncRNA MT1JP overexpression on proliferation of GC cell line. b, c Effect of MiR-92a-3p mimics, si-FBXW7, and IncRNA MT1JP overexpression on apoptosis of GC cell line. d-e Effect of MiR-92a-3p mimics, si-FBXW7, and InCRNA MT1JP on migration and invasion of GC cell. Effect of miR-92a-3p mimics, si-FBXW7, and InCRNA MT1JP on proliferation of SGC7901 (f) and MGC-823 (g)

showed that a significantly positive correlation between lncRNA MT1JP and FBXW7 expression was observed in GC tissues. Moreover, western blotting analysis also showed that miR-92a-3p mimic suppressed the expression of FBXW7, whereas IncRNA MT1JP overexpression substantially abolished this effect. Besides, although MT1JP was overexpressed, FBXW7 expression was not remarkably altered when knocked down miR-92a-3p. These results supported that lncRNA MT1JP involved in post-transcriptional regulation of FBXW7 by sponging miR-92a-3p.
Mounting evidence indicated that miR-92a-3p was often dysregulated in GC, and expression of miR-92a was remarkably related to the development of GC and had a prognosis value for GC $[25,26]$. In this study, compared with negative control, miR-92a-3p significantly increased cell proliferation, migration and invasion and inhibited cell apoptosis in BGC-823 cell. Intriguing, in line with our study, miR-92a-3p was reported to promote tumor growth in multiple tumors by targeting FBXW7 [27-29]. FBXW7 is a member of 
SCF (complex of SKP1, CUL1 and F-box protein)-type ubiquitin ligase complex, which regulate target protein ubiquitination and degradation. The substrates of FBXW7 include several broadly investigated oncoproteins, such as MYC, cyclin E, mTOR and Notch. It is well known that FBXW7 is a tumor suppressor and its down-regulation was displayed in numerous human malignancies, including GC [30-32]. FBXW7 was reported to regulate tumor apoptosis, growth arrest and epithelial-to-mesenchymal transition in GC, and it also played an important role in drug resistance $[33,34]$. The E-cadherin and MT1JP acted as the key proteins in the epithelial-to-mesenchymal transition. We use The Cancer Genome Atlas(TCGA) to explore the association between the FSP1and E-cadherin and MT1JP. The results indicated the expression of FSP1and E-cadherin were significantly associated with the expression of MT1JP (Additional file 1: Figure S10), which suggested MT1JP may play important role in epithelial-to-mesenchymal transition. In the present study, we have also shown that lncRNA MT1JP regulate FBXW7 expression by competition for miR-92a-3p binding. Up-regulated lncRNA MT1JP may lead to increase the expression of FBXW7, and consequently inhibited cell proliferation and promoted cell apoptosis in GC. These changes in GC cells could be reversed by miR-92a mimics and si-FBXW7 vector, according to rescue experiments in vitro.

\section{Conclusions}

In summary, we identified MT1JP, a significantly downregulated lncRNA, was associated with advance stage of GC, and GC patients with higher expression of MT1JP had a poorer survival, suggesting a promising prognostic role of MT1JP in GC. Functional analysis suggested that lncRNA MT1JP overexpression inhibited cell proliferation, migration, invasion and promoted cell apoptosis. Rescue assays suggested up-regulated miR-92a-3p and downregulated FBXW7 reversed cell phenotypes caused by lncRNA MT1JP. Our study provided new insight into the post-transcriptional regulation mechanism of lncRNA MT1JP involved in the development of GC.

\section{Additional file}

Additional file 1: Figure S1-S10 (Online). Table S1. The top 10 significantly upregulated and downregulated IncRNAs identified by Arraystar Human IncRNA/mRNA chip. Table S2. The prime sequences of target genes used in real-time PCR. (DOCX 1257 kb)

\section{Abbreviations}

ceRNA: competing endogenous RNA; GC: Gastric cancer; GEO: Gene Expression Omnibus; IncRNA: long noncoding RNA; MT1JP: Metallothionein $1 \mathrm{~J}$, pseudogene; qRT-PCR: Quantitative reverse transcription polymerase chain reaction; TCGA: The Cancer Genome Atlas

\section{Acknowledgments}

This study was partly supported by National Natural Science Foundation of China (81473049, 81230068, 81773539, and 81302490), Jiangsu Provincial Science and Technology Innovation Team, Jiangsu Provincial Postdoctoral Science Foundation funded project (1501081C), China Postdoctoral Science Foundation funded project (2015 M580449), Collaborative Innovation Center For Cancer Personalized Medicine, and the Priority Academic Program Development of Jiangsu Higher Education Institutions (Public Health and Preventive Medicine).

\section{Authors' contributions}

$Z G, Z Z$, and ZA designed and performed the research. WQ, ZQ, WD, GW, CH, and WM collected data. DM, MG, and GY performed statistical analysis. ZG, LS and LJ wrote the draft manuscript. All authors contributed to the writing and reviewing of the manuscript, and approved the final manuscript for submission.

Ethics approval and consent to participate

The research was approved by the Ethics Committee of Nanjing Medical University.

Consent for publication

All subjects have written informed consent.

\section{Competing interests}

The authors declare that they have no competing interests.

\section{Publisher's Note}

Springer Nature remains neutral with regard to jurisdictional claims in published maps and institutional affiliations.

\section{Author details}

'Department of Neurology, Children's Hospital of Nanjing Medical University, Nanjing, China. ${ }^{2}$ Department of Environmental Genomics, School of Public Health, Jiangsu Key Laboratory of Cancer Biomarkers, Prevention and Treatment, Collaborative Innovation Center for Cancer Personalized Medicine, Nanjing Medical University, 101 Longmian Avenue, Jiangning District, Nanjing 211166, China. ${ }^{3}$ Department of Genetic Toxicology, The Key Laboratory of Modern Toxicology of Ministry of Education, School of Public Health, Nanjing Medical University, Nanjing, China. ${ }^{4}$ Department of General Surgery, The Second Affiliated Hospital of Nanjing Medical University, Nanjing, China. ${ }^{5}$ Department of General Surgery, Yixing Cancer Hospital, Yixing, China. ${ }^{6}$ Key Laboratory of Environmental Pollution Monitoring and Disease Control, Ministry of Education, Guizhou Medical University, Guiyang 550025, Guizhou, China.

Received: 13 August 2017 Accepted: 29 March 2018

Published online: 02 May 2018

References

1. Djebali S, et al. Landscape of transcription in human cells. Nature. 2012;489: $101-8$.

2. Huarte M. The emerging role of IncRNAs in cancer. Nat Med. 2015;21:1253-61.

3. Esteller M. Non-coding RNAs in human disease. Nat Rev Genet. 2011;12: 861-74.

4. Schmitt AM, Chang HY. Long noncoding RNAs in Cancer pathways. Cancer Cell. 2016;29:452-63.

5. Khalil AM, et al. Many human large intergenic noncoding RNAs associate with chromatin-modifying complexes and affect gene expression. Proc Natl Acad Sci U S A. 2009;106:11667-72.

6. Lee JT. Epigenetic regulation by long noncoding RNAs. Science. 2012;338: 1435-9.

7. Rinn JL, Chang HY. Genome regulation by long noncoding RNAs. Annu Rev Biochem. 2012;81:145-66.

8. Thomson DW, Dinger ME. Endogenous microRNA sponges: evidence and controversy. Nat Rev Genet. 2016;17:272-83.

9. Cesana $\mathrm{M}$, et al. A long noncoding RNA controls muscle differentiation by functioning as a competing endogenous RNA. Cell. 2011;147:358-69.

10. Lu MH, et al. Long noncoding RNA BC032469, a novel competing endogenous RNA, upregulates hTERT expression by sponging miR-1207-5p and promotes proliferation in gastric cancer. Oncogene. 2016;35:3524-34. 
11. Ferlay J, et al. Cancer incidence and mortality worldwide: sources, methods and major patterns in GLOBOCAN 2012. Int J Cancer. 2015;136:E359-86.

12. Soerjomataram I, et al. Global burden of cancer in 2008: a systematic analysis of disability-adjusted life-years in 12 world regions. Lancet. 2012; 380:1840-50.

13. Liang $\mathrm{H}$, Kim YH. Identifying molecular drivers of gastric cancer through next-generation sequencing. Cancer Lett. 2013;340:241-6.

14. Fang XY, Pan HF, Leng RX, Ye DQ. Long noncoding RNAs: novel insights into gastric cancer. Cancer Lett. 2015;356:357-66.

15. Chandra, Gupta S, Nandan Tripathi Y. Potential of long non-coding RNAs in cancer patients: from biomarkers to therapeutic targets. Int J Cancer. 2017; 140(9):1955-967

16. Xue $Y$, et al. Genome-wide analysis of long noncoding RNA signature in human colorectal cancer. Gene. 2015;556:227-34.

17. Wang $L$, et al. CPAT: coding-potential assessment tool using an alignmentfree logistic regression model. Nucleic Acids Res. 2013;41:e74.

18. Wang S, et al. PSCA rs2294008 polymorphism contributes to the decreased risk for cervical cancer in a Chinese population. Sci Rep. 2016;6:23465.

19. Chu H, et al. Identification of novel piRNAs in bladder cancer. Cancer Lett. 2015;356:561-7.

20. Sun TT, et al. LncRNA GClnc1 promotes gastric carcinogenesis and may act as a modular scaffold of WDR5 and KAT2A complexes to specify the histone modification pattern. Cancer Discov. 2016;6:784-801.

21. Liu $L$, et al. LncRNA MT1JP functions as a tumor suppressor by interacting with TIAR to modulate the p53 pathway. Oncotarget. 2016;7:15787-800.

22. Hu Y, Tian H, Xu J, Fang JY. Roles of competing endogenous RNAs in gastric cancer. Brief Funct Genomics. 2016;15:266-73.

23. Xia T, et al. Long noncoding RNA associated-competing endogenous RNAs in gastric cancer. Sci Rep. 2014;4:6088.

24. Liu XH, et al. Lnc RNA HOTAIR functions as a competing endogenous RNA to regulate HER2 expression by sponging miR-331-3p in gastric cancer. Mol Cancer. 2014;13:92.

25. Ren $C$, et al. Expression and prognostic value of miR-92a in patients with gastric cancer. Tumour Biol. 2016;37:9483-91.

26. Wu Q, et al. MiR-19b/20a/92a regulates the self-renewal and proliferation of gastric cancer stem cells. J Cell Sci. 2013;126:4220-9.

27. Yang W, et al. MicroRNA-92a contributes to tumor growth of human hepatocellular carcinoma by targeting FBXW7. Oncol Rep. 2015;34:2576-84.

28. Zhou C. et al. miR-92a is upregulated in cervical cancer and promotes cell proliferation and invasion by targeting FBXW7. Biochem Biophys Res Commun. 2015;458:63-9.

29. Liu C, Zhang Y, Chen H, Jiang L, Xiao D. Function analysis of rs9589207 polymorphism in miR-92a in gastric cancer. Tumour Biol. 2016;37:4439-44.

30. Welcker M, Clurman BE. FBW7 ubiquitin ligase: a tumour suppressor at the crossroads of cell division, growth and differentiation. Nat Rev Cancer. 2008; 8:83-93.

31. Davis RJ, Welcker M, Clurman BE. Tumor suppression by the Fbw7 ubiquitin ligase: mechanisms and opportunities. Cancer Cell. 2014;26:455-64.

32. Akhoondi S, et al. FBXW7/hCDC4 is a general tumor suppressor in human cancer. Cancer Res. 2007;67:9006-12.

33. Li H, et al. Fbxw7 regulates tumor apoptosis, growth arrest and the epithelial-to-mesenchymal transition in part through the RhoA signaling pathway in gastric cancer. Cancer Lett. 2016;370:39-55.

34. Eto $\mathrm{K}$, et al. The sensitivity of gastric cancer to trastuzumab is regulated by the miR-223/FBXW7 pathway. Int J Cancer. 2015;136:1537-45.

\section{Submit your next manuscript to BioMed Central and we will help you at every step:}

- We accept pre-submission inquiries

- Our selector tool helps you to find the most relevant journal

- We provide round the clock customer support

- Convenient online submission

- Thorough peer review

- Inclusion in PubMed and all major indexing services

- Maximum visibility for your research

Submit your manuscript at www.biomedcentral.com/submit
Biomed Central 